\title{
ON A BOUNDARY VALUE PROBLEM ARISING IN ELASTIC DEFLECTION THEORY
}

\author{
XIUQIN WANG
}

\begin{abstract}
In this paper, a finite-difference method for the determination of an approximate solution of a fourth-order two-point boundary value problem is presented under the nonresonance condition. The solution of this linear problem can be used to find approximate solutions of a broad range of nonlinear problems in applications.
\end{abstract}

\section{INTRODUCTION}

It is well known that the fourth-order two-point linear boundary problem

$$
\begin{aligned}
y^{(4)}-F(x) y & =G(x), \quad a<x<b, \\
y(a) & =A_{0}, \quad y(b)=A_{1}, \quad y^{\prime \prime}(a)=B_{0}, \quad y^{\prime \prime}(b)=B_{1},
\end{aligned}
$$

where $F$ and $G$ are real-valued continuous functions on the closed interval $[a, b]$ and $A_{0}, B_{0}, A_{1}, B_{1}$ are real constants, occurs frequently in elastic deflection theory. A straightforward substitution $X=(x-a) /(b-a)$ transforms the above problem into the following problem of the standard type

$$
\begin{aligned}
y^{(4)}-f(x) y & =g(x), \quad 0<x<1, \\
y(0) & =a_{0}, \quad y(1)=a_{1}, \quad y^{\prime \prime}(0)=b_{0}, \quad y^{\prime \prime}(1)=b_{1},
\end{aligned}
$$

where the new independent variable is still denoted by $x$ for convenience. Note that the difference between the second-order problems and the fourth-order problems is that in the latter situation important tools such as the maximum principle are often unavailable.

In general, (1.1) cannot be solved in closed form and numerical methods are to be resorted to. In [13], Usmani and Marsden gave a finite-difference method of order $\mathrm{O}\left(h^{2}\right)$ under the condition

$$
-36 \leqslant f(x) \leqslant 0 \text { for } x \in[0,1] .
$$

\footnotetext{
Received 22nd March, 2006
}

The author thanks the referee for helpful suggestions which improved the presentation of this paper.

Copyright Clearance Centre, Inc. Serial-fee code: 0004-9727/06 \$A2.00+0.00. 
Afterwards, under a similar condition, methods of orders of $O\left(h^{4}\right)$ and $O\left(h^{6}\right)$ were derived by Jain, Iyengar, and Saldanha [4] and Usmani [9]. Later in [14], an $O\left(h^{2}\right)$ method was obtained by Usmani and Marsden under the hypothesis

$$
f(x) \leqslant 0, \quad x \in[0,1],
$$

which is much less restrictive than the condition (1.2). Attempts have been made to further relax the condition (1.3). In [10, 11], Usmani proved that problem (1.1) has a unique solution for arbitrarily given $f, g, a_{0}, b_{0}, a_{1}, b_{1}$ provided that $f$ satisfies

$$
|f(x)|<\pi^{4} \text { for } x \in[0,1] \text {. }
$$

Besides, Usmani [11] also obtained a finite difference scheme of order $\mathrm{O}\left(h^{\mathbf{3} 2}\right)$ (in $[10,11]$, the condition was stated in a weaker form $f(x)<\pi^{4}$ but his proof worked only [12] for $f$ satisfying (1.4)). Later, Yang [17] proved that (1.1) has a unique solution under the general nonresonance condition

$$
f(x) \neq j^{4} \pi^{4}, \quad j=1,2, \ldots, \quad x \in[0,1],
$$

which motivates us to ask the question whether a finite-difference approximation method can be carried out for (1.1) under this same general condition.

Indeed, our aim of the present paper is to give a uniform treatment of the boundary value problem (1.1) under the general condition (1.5) imposed on the function $f$. Note that if $f(x) \equiv j^{4} \pi^{4}$ for some positive integer $j$, then the problem (1.1) is not solvable in general. For instance, if $f(x) \equiv \pi^{4}$, then (1.1) has no solution for $g(x) \equiv 0$ and $a_{0}+a_{1}-\left(b_{0}+b_{1}\right) / \pi^{2} \neq 0$. Thus, our condition is quite general.

The finite-difference method we shall obtain below is of the order $\mathrm{O}\left(h^{3 / 2}\right)$. This means that we obtain the generality of our method at the sacrifice of high convergence rate. Such a reality has already been witnessed by various earlier studies of similar problems, in particular, by the studies of (1.1) of various authors cited above. Note also that, in the $h \rightarrow 0$ limit, we recover the classical solution of (1.1), thus providing an alternative (discretised) proof of the main existence theorem in [17]. In a recent work [8], Thompson and Tisdell study the finite-difference methods for approximating solutions of some boundary value problems of systems of second-order equations, which may be applied to higher-order equations.

In the next section, we introduce our finite-difference approximation scheme for the boundary value problem (1.1) and state our main convergence theorem in general terms. In Section 3, we carry out a detailed analysis of the finite-difference scheme and estimate the error bounds with respect to various parameters in the problem. We then give a precise statement of the convergence theorem. In Section 4, we comment on some applications and discuss future directions. 


\section{Finite Difference Scheme and Convergence THEOREM}

Let $N$ be a positive integer and, for convenience, set

$$
(h) \equiv\{x \in[0,1]: x=j h, j=1,2, \ldots, N\}
$$

where $h \equiv(N+1)^{-1}$. The set of all real-valued functions $u$ defined on $(h)$ is a real vector space of dimension $N$ which can be identified with the Euclidean space $\mathbb{R}^{N}$. We shall denote the usual inner product of $\mathbb{R}^{N}$ by $(\cdot, \cdot)$ and the associated norm by $\|\cdot\|$ where $\|u\|=(u, u)^{1 / 2}$. Note that for $u, v \in \mathbb{R}^{N}$,

$$
(u, v)=u^{\tau} v=\sum u\left(x_{j}\right) v\left(x_{j}\right)=\sum u_{j} v_{j}
$$

where $u^{\tau}$ denotes the usual transpose of the column vector $u=\left(u\left(x_{j}\right)\right) \equiv\left(u_{j}\right)$. We shall also apply the operation $\tau$ on matrices.

We now discretise the problem (1.1) by the following (central) finite-difference scheme (see also $[11,13,14]$ )

$$
\begin{aligned}
-2 y_{0}+5 y_{1}-4 y_{2}+y_{3} & =-h^{2} y_{0}^{\prime \prime}+h^{4}\left(-\frac{1}{12} y_{0}^{(4)}+y_{1}^{(4)}\right)+t_{1}, \\
y_{j-2}-4 y_{j-1}+6 y_{j}-4 y_{j+1}+y_{j+2} & =h^{4} y_{j}^{(4)}+t_{j}, \quad j=2, \ldots, N-1, \\
y_{N-2}-4 y_{N-1}+5 y_{N}-2 y_{N+1} & =-h^{2} y_{N+1}^{\prime \prime}+h^{4}\left(y_{N}^{(4)}-\frac{1}{12} y_{N+1}^{(4)}\right)+t_{N},
\end{aligned}
$$

where $y_{j}=y\left(x_{j}\right), x_{j}=j h, j=0,1,2, \ldots, N+1$, and the truncation errors of (2.1) are

$$
\begin{aligned}
t_{1} & =\frac{59}{360} h^{6} y^{(6)}\left(\tilde{x}_{1}\right), \quad x_{0}<\tilde{x}_{1}<x_{3}, \\
t_{j} & =\frac{1}{6} h^{6} y^{(6)}\left(\widetilde{x}_{j}\right), \quad x_{j-2}<\widetilde{x}_{j}<x_{j+2}, \quad j=2, \ldots, N-1, \\
t_{N} & =\frac{59}{360} h^{6} y^{(6)}\left(\widetilde{x}_{N}\right), \quad x_{N-2}<\widetilde{x}_{N}<x_{N+1} .
\end{aligned}
$$

On substituting (2.1) into the discrete version of the differential equation in (1.1), namely,

$$
y^{(4)}\left(x_{j}\right)=f\left(x_{j}\right) y\left(x_{j}\right)+g\left(x_{j}\right), \quad j=1,2, \ldots, N,
$$

we obtain the matrix equation

$$
P^{2} Y=h^{4} D Y+C+T
$$

where $P$ is the $N \times N$ tridiagonal matrix defined by

$$
P=\left(\begin{array}{ccccccc}
2 & -1 & 0 & \ldots & \ldots & \ldots & 0 \\
-1 & 2 & -1 & \ldots & \ldots & \ldots & 0 \\
0 & -1 & 2 & -1 & \ldots & \ldots & 0 \\
\cdots & \ldots & \ldots & \ldots & \ldots & \ldots & \ldots \\
0 & \ldots & \ldots & \ldots & -1 & 2 & -1 \\
0 & \ldots & \ldots & \ldots & 0 & -1 & 2
\end{array}\right)
$$


(see $[11,13,14]), Y=\left(y_{j}\right), D=\operatorname{diag}\left(f\left(x_{j}\right)\right) \equiv \operatorname{diag}\left(f_{j}\right)$ is an $N \times N$ diagonal matrix, $T=\left(t_{j}\right)$, and $C=\left(c_{j}\right)$ is determined by

$$
\begin{aligned}
c_{1} & =g_{1} h^{4}+2 a_{0}-b_{0} h^{2}-\frac{h^{4}}{12}\left(f_{0} a_{0}+g_{0}\right), \\
c_{2} & =g_{2} h^{4}-a_{0}, \\
c_{j} & =g_{j} h^{4}, \quad j=3,4, \ldots, N-2, \\
c_{N-1} & =g_{N-1} h^{4}-a_{1}, \\
c_{N} & =g_{N} h^{4}+2 a_{1}-b_{1} h^{2}-\frac{h^{4}}{12}\left(f_{N+1} a_{1}+g_{N+1}\right) .
\end{aligned}
$$

We can state our main convergence theorem as follows.

THEOREM 2.1. The discrete equation

$$
P^{2} Y=h^{4} D Y+C
$$

obtained from the finite-difference scheme (2.1) for the two-point boundary value problem (1.1) always has a unique solution when $h>0$ is sufficiently small and $f(x)$ satisfies the condition (1.5) and such a solution approximates the unique solution of (1.1) at the mesh points $x_{j}=j h(j=1,2, \ldots, N)$ with an error of the order $O\left(h^{3 / 2}\right)$.

We shall present a precise statement of the above theorem in the next section after a detailed analysis.

\section{Convergence Analysis}

Since $f(x)$ is continuous on the interval $[0,1]$ and $f(x) \neq j^{4} \pi^{4}$ for $j=1,2, \ldots$, if we define $p=\inf f(x)$ and $q=\sup f(x)$, then either

$$
q<\pi^{4}
$$

or there is an integer $k$ such that

$$
k^{4} \pi^{4}<p \leqslant q<(k+1)^{4} \pi^{4} .
$$

It is well known that the eigenvalues of $P$ are $4 \sin ^{2}(j \pi h / 2), j=1,2, \ldots, N$. Therefore the eigenvalues $\lambda_{j}$ of $P^{2}$ are

$$
\lambda_{j}=16 \sin ^{4}\left(\frac{j \pi h}{2}\right), \quad j=1,2, \ldots, N .
$$

Note also that the standard calculus inequality $t-t^{3} / 6<\sin t<t, t \in(0, \pi / 2)$, gives us the estimates

$$
j^{4} \pi^{4} h^{4}\left(1-\frac{j^{2} \pi^{2} h^{2}}{24}\right)^{4}<\lambda_{j}<j^{4} \pi^{4} h^{4}, \quad j=1,2, \ldots, N .
$$


Choose an orthogonal $N \times N$ matrix $O$ such that

$$
O^{\top} P^{2} O=\operatorname{diag}\left(\lambda_{1}, \lambda_{2}, \ldots, \lambda_{N}\right)
$$

and introduce a substitution $\widetilde{U}=O^{\top} U$ where $U, \widetilde{U} \in \mathbf{R}^{N}$. We have

$$
\begin{aligned}
\left(U,\left(P^{2}-h^{4} D\right) U\right) & =U^{\tau} P^{2} U-h^{4} U^{\tau} D U \\
& \geqslant U^{\tau} P^{2} U-q h^{4} U^{\tau} U \\
& =\tilde{U}^{\tau} \operatorname{diag}\left(\lambda_{1}, \lambda_{2}, \ldots, \lambda_{N}\right) \tilde{U}-q h^{4} \tilde{U}^{\tau} \tilde{U} \\
& \geqslant\left(\lambda_{1}-q h^{4}\right) \tilde{U}^{\tau} \tilde{U} \\
& =\left(\lambda_{1}-q h^{4}\right)(U, U) .
\end{aligned}
$$

But, from (3.4), we have $\lambda_{1}>\pi^{4} h^{4}\left(1-\pi^{2} h^{2} / 24\right)^{4}$. Consequently

$$
\left(U,\left(P^{2}-h^{4} D\right) U\right) \geqslant h^{4}\left(\pi^{4}\left[1-\frac{\pi^{2} h^{2}}{24}\right]^{4}-q\right)(U, U) .
$$

Now assume $f(x)$ satisfies (3.1) and let

$$
h \leqslant \frac{2 \sqrt{6}}{\pi}\left(1-\left[\frac{1}{2}+\frac{q}{2 \pi^{4}}\right]^{1 / 4}\right)^{1 / 2}
$$

From (3.7) and (3.8), we obtain

$$
\left(U,\left(P^{2}-h^{4} D\right) U\right) \geqslant \frac{h^{4}}{2}\left(\pi^{4}-q\right)(U, U) .
$$

It then yields from (3.9) and the Schwartz inequality that $P^{2}-h^{4} D$ is invertible when $h$ satisfies (3.8), and that

$$
\left\|\left(P^{2}-h^{4} D\right)^{-1} U\right\| \leqslant \frac{2}{h^{4}\left(\pi^{4}-q\right)}\|U\|, \quad U \in \mathbb{R}^{N} .
$$

If $f(x)$ satisfies (3.2), we assume for simplicity that $N \geqslant k$. Set $m=(p+q) / 2$. Then

$$
\begin{aligned}
\left\|\left(P^{2}-h^{4} m I\right) U\right\|^{2} & =\widetilde{U}^{\tau} O^{\tau}\left(P^{2}-h^{4} m I\right)^{2} O \tilde{U} \\
& =\sum \tilde{U}_{j}^{2}\left(\lambda_{j}-h^{4} m\right)^{2} .
\end{aligned}
$$

From (3.11), we have

$$
\begin{aligned}
\left\|\left(P^{2}-h^{4} D\right) U\right\| & \geqslant\left\|\left(P^{2}-h^{4} m I\right) U\right\|-\left\|\left(h^{4} m I-h^{4} D\right) U\right\| \\
& \geqslant\|\widetilde{U}\| \min _{j}\left|\lambda_{j}-h^{4} m\right|-h^{4}\|U\| \max _{j}\left|m-f_{j}\right| .
\end{aligned}
$$

It is easily checked that if

$$
h<h_{1} \equiv \frac{2 \sqrt{6}}{(k+1) \pi}\left(1-\frac{m^{1 / 4}}{(k+1) \pi}\right)^{1 / 2}
$$


then

$$
m<(k+1)^{4} \pi^{4}\left(1-\frac{(k+1)^{2} \pi^{2} h^{2}}{24}\right)^{4}
$$

and if

$$
h<h_{2} \equiv \frac{2 \sqrt{6}}{(k+1) \pi}\left(1-\left[\frac{1}{2}+\frac{q}{2(k+1)^{4} \pi^{4}}\right]^{1 / 4}\right)^{1 / 2},
$$

then

$$
(k+1)^{4} \pi^{4}\left(1-\frac{(k+1)^{2} \pi^{2} h^{2}}{24}\right)^{4}-m-\frac{(q-p)}{2}>\frac{1}{2}\left([k+1]^{4} \pi^{4}-q\right) .
$$

From (3.14) and (3.16), it follows that if $h<h_{0} \equiv \min \left(h_{1}, h_{2}\right)$, then (3.12) gives us

$$
\left\|\left(P^{2}-h^{4} D\right) U\right\| \geqslant h^{4} d_{0}\|U\|
$$

where

$$
d_{0} \equiv \min \left(p-k^{4} \pi^{4}, \frac{1}{2}\left([k+1]^{4} \pi^{4}-q\right)\right) .
$$

From the above analysis, we see clearly that if

$$
h<h_{0} \text {, }
$$

the matrix $P^{2}-h^{4} D$ is invertible and satisfies the bound

$$
\left\|\left(P^{2}-h^{4} D\right)^{-1} U\right\| \leqslant \frac{1}{h^{4} d_{0}}\|U\|, \quad U \in \mathbb{R}^{N},
$$

which is the key estimate to our error estimate.

On neglecting the truncation error term $T$ in the equation (2.4), we arrive at the truncated equation

$$
P^{2} Z=h^{4} D Z+C,
$$

where the solution vector $Z=\left(z_{j}\right)$ is viewed as an approximate solution of the (exact) equation (2.4). For any data term $C=\left(c_{j}\right)$, the equation (3.21) has a unique solution for sufficiently small $h$ because we can choose $h$ to satisfy (3.8) or (3.19) according to $f(x)$ satisfying (3.1) or (3.2) to make $\left(P^{2}-h^{4} D\right)$ invertible.

Let $E=Y-Z=\left(y\left(x_{j}\right)-z_{j}\right)$ where $y(x)$ and $Z$ are the unique solutions of (1.1) and (3.21), respectively. It is seen that (the error vector) $E \equiv\left(e_{j}\right)$ in fact is a solution of the equation

$$
P^{2} E=h^{4} D E+T
$$


Hence, from (3.10) or (3.20) according to $f(x)$ satisfying (3.1) or (3.2), we obtain

$$
\begin{aligned}
\|E\| & \leqslant\left\|\left(P^{2}-h^{4} D\right)^{-1} T\right\| \\
& \leqslant h^{-4} M\|T\|,
\end{aligned}
$$

where $M$ is a constant depending only on $f(x)$. In particular, (3.23) implies the following pointwise bound

$$
\begin{aligned}
\max _{j}\left|e_{j}\right| & \leqslant\|E\| \\
& \leqslant h^{-4} M N^{1 / 2} \max _{j}\left|t_{j}\right| \\
& \leqslant h^{-9 / 2} M \max _{j}\left|t_{j}\right| .
\end{aligned}
$$

Combining (2.2) and (3.24), we see that we can conclude with

THEOREM 3.1. Suppose that $f(x) \neq j^{4} \pi^{4}$ for $j=1,2, \ldots$ and that $y(x)$ is the unique solution of the fourth-order two-point boundary value problem (1.1) which has continuous derivatives up to the 6th order. Let (3.21) be the matrix equation for the unknown vector $Z=\left(z_{j}\right)$ which is the truncated discrete equation derived from the finite-difference scheme (2.1) or (2.4). If the step size $h$ is sufficiently small such that it falls into the range (3.19), then the equation (3.21) is uniquely solvable and this solution pointwise approximates the exact unique solution of (1.1) according to the error bound

$$
\max _{j}\left|y\left(x_{j}\right)-z_{j}\right|=O\left(h^{3 / 2}\right) .
$$

It is seen that the above theorem is the precise form of Theorem 2 .

\section{Comments on Applications}

The boundary value problem (1.1) studied in this paper is a prototype problem in elastic theory (the beam or plate deflection problem) and its extensions to other situations can be carried out following a similar path for which a crucial technical restriction is the nonresonance condition (1.5). For example, the boundary conditions in (1.1) and the terms of the diffrential equation in (1.1) can all be modified for application purposes. Besides, we can also extend this work to include for example finite-element approximations $[1,2,3,18]$, which will be addressed elsewhere.

Our method can be applied to the study of some nonlinear problems $[6,16,17]$ which arise frequently in many areas of engineering. For example, in the study of the design and performance of microelectromechanical systems (MEMS), one encounters the 
equations of the forms

$$
\begin{aligned}
y^{(4)}-f(x) y & =\frac{\lambda g(x)}{(1+h(x)-y)^{2}}, \\
y^{(4)}+a y^{\prime \prime}-f(x) y & =\frac{\lambda g(x)}{1+b \int_{0}^{1} y^{2}(x) \mathrm{d} x}, \\
y^{(4)}-f(x) y & =\frac{\lambda g(x)}{(1+y)^{2}\left(1+a \int_{0}^{1}(1+y)^{-1}(x) \mathrm{d} x\right)^{2}},
\end{aligned}
$$

subject to various kinds of boundary conditions of elastic deformation problem nature (see $[5,7,15,19]$ for example). The parameter $\lambda$ represents the magnitude of an applied electric voltage which is usually assumed to be small (a large value of $\lambda$ breaks the device and solution will no longer exist). One is interested in small amplitude solutions. For these problems, we discretise the equations as before and view the right-hand sides of (4.1)-(4.3) as the nonhomogeneous term in (1.1). In the discretised problems, these give rise to the vector $C$ in (3.21) and lead to a nonlinear equation of the form

$$
P^{2} Z=h^{4} D Z+C(Z) .
$$

Under the nonresonance condition, an operator bound of the form (3.10) or (3.20) can be used to show that when the parameter $\lambda$ is small, (4.4) may be solved iteratively by the scheme

$$
P^{2} Z_{n}=h^{4} D Z_{n}+C\left(Z_{n-1}\right), \quad n=1,2, \ldots, \quad Z_{0}=0,
$$

whose convergence is ensured by the contraction mapping principle. Obviously, as $h \rightarrow 0$, the obtained solution approaches a solution of the original continuous problem.

\section{REFERENCES}

[1] P. Bochev and R.B. Lehoucq, 'On the finite element solution of the pure Neumann problem', SIAM Rev. 47 (2005), 50-66.

[2] Z. Chen, Finite element methods and their applications (Springer-Verlag, Berlin, 2005).

[3] G.J. Fix and J.P. Roop, 'Least squares finite-element solution of a fractional order two-point boundary value problem', Comput. Math. Appl. 48 (2004), 1017-1033.

[4] M.K. Jain, S.R.K. Iyengar and J.S.V. Saldanha, 'Numerical solution of a fourth-order ordinary differential equation', J. Engrg. Math. 11 (1977), 373-380.

[5] R. Legtenberg, J. Gilbert, S.D. Senturia and M. Elwenspoek, 'Electrostatic curved electrode actuators', J. Microelectromechanical Sys. 6 (1997), 257-265.

[6] R. Ma, J. Zhang and S. Fu, 'The method of lower and upper solutions for fourth-order two-point boundary value problems', J. Math. Anal. Appl. 215 (1997), 415-422.

[7] J.A. Pelesko and D.H. Bernstein, Modeling MEMS and NEMS (Chapman and Hall, New York, 2003). 
[8] H.B. Thompson and C. Tisdell, 'Systems of difference equations associated with boundary value problems for second order systems of ordinary differential equations', J. Math. Anal. Appl. 248 (2000), 333-347.

[9] R.A. Usmani, 'An $\mathrm{O}\left(h^{6}\right)$ finite difference analogue for the solution of some differential equations occuring in plate deflection theory', J. Inst. Math. Appl. 20 (1977), 331-333.

[10] R.A. Usmani, 'Discrete methods for boundary value problems with applications in plate deflection theory', Z. Angew. Math. Phys. 30 (1979), 87-99.

[11] R.A. Usmani, 'A uniqueness theorem for a boundary value problem', Proc. Amer. Math. Soc. (1979), 329-335.

[12] R.A. Usmani, Private correspondence.

[13] R.A. Usmani and M.J. Marsden, 'Numerical solution of some ordinary differential equations occuring in plate deflection theory', J. Engrg. Math. 9 (1975), 1-10.

[14] R.A. Usmani and M.J. Marsden, 'Convergence of a numerical procedure for the solution of a fourth-order boundary value problem', Proc. Indian Acad. Sci. Sect. A. Math. Sci 88 (1979), 21-30.

[15] P.K.C. Wang and F.Y. Hadaegh, 'Computation of static shapes and voltages for micromachined deformable mirrors with nonlinear electrostatic actuators', J. Microelectromechanical Sys. 5 (1996), 205-220.

[16] Y.-M. Wang, 'On fourth-order elliptic boundary value problems with nonmonotone nonlinear function', J. Math. Anal. Appl. 307 (2005), 1-11.

[17] Y. Yang, 'On fourth-order two-point boundary value problems', Proc. Amer. Math. Soc. 104 (1988), 175-180.

[18] Y. Yang, 'Convergence of the pseudospectral method for the Ginzburg-Landau equation', J. Math. Anal. Appl. 147 (1990), 556-568.

[19] M.I. Younis, E.M. Abdel-Rahman and A. Nayfeh, 'A reduced-order model for electrically actuated microbeam-based MEMS', J. Microelectromechanical Sys. 12 (2003), 672-280.

School of Mathematics

Henan University

Kaifeng

Henan 475001

Peoples Republic of China

e-mail: xiuqinwang@henu.edu.cn 